\title{
La regulación de etiquetado en la industria del tabaco de México: efecto de los pictogramas en la demanda de tabaco ${ }^{1}$
}

\author{
Labeling Regulation in the Mexican Tobacco Industry: \\ Effect of Pictorial Warning Labels on Tobacco Demand \\ Jorge Ibarra Salazar, ${ }^{2}$ César González Caloca, ${ }^{3}$ Silvia Núñez Gómez, ${ }^{4}$ Manuel Ramírez García, ${ }^{5}$ \\ Mario Rodríguez Nuncio ${ }^{6}$ y Catalina Santos González ${ }^{7}$
}

\begin{abstract}
RESUMEN
En este artículo se analiza el efecto de las advertencias sanitarias con pictogramas en la demanda de tabaco de México. Se estiman los modelos de demanda convencional, miope y racional con una serie de tiempo trimestral de 1994 a 2013, usando mínimos cuadrados ordinarios y mínimos cuadrados en dos etapas con variables instrumentales. Se aplica el método de Prais-Winsten para corregir autocorrelación y se estiman errores robustos para corregir heteroscedasticidad. Se encontró evidencia de que la segunda y tercera rondas de pictogramas causaron una reducción significativa en el consumo de aproximadamente una cajetilla de cigarros per cápita. La política regulatoria de empaquetado y etiquetado de cajetillas de cigarro ha sido efectiva para reducir la demanda de tabaco en México.
\end{abstract}

Palabras clave: regulación industria tabaco, pictogramas, demanda de tabaco, México. Clasificación JEL: I18, L66, L51, C22.

\footnotetext{
Abstract

In this paper, we investigate the effect of graphic warning labels on the Mexican tobacco demand. The conventional, myopic and rational models of demand are estimated with quarterly time series from 1994 to 2013 . We use ordinary least squares and two-stage least squares with instrumental variables. We used the Prais-Winsten method to correct autocorrelation and estimated robust errors to correct heteroscedasticity. We found evidence that the second and third pictograms rounds caused a significant reduction in the

1 Fecha de recepción: 17/02/2017. Fecha de aprobación: 11/04/2019.

2 Tecnológico de Monterrey, México. Correo electrónico: jaibarra@itesm.mx. ORCID: 0000-0002-9581-9099.

3 Tecnológico de Monterrey, México. Correo electrónico: cesar.glz.caloca@hotmail.com. ORCID: 0000-0002-29638353.

4 Tecnológico de Monterrey, México. Correo electrónico: gnunez.silvia@gmail.com. ORCID: 0000-0002-5134-4191.

5 Tecnológico de Monterrey, México. Correo electrónico: rmanueel@gmail.com. ORCID: 0000-0001-8206-2413.

6 Tecnológico de Monterrey, México. Correo electrónico: mario.rdnu@gmail.com. ORCID: 0000-0003-0552-2791.

7 Tecnológico de Monterrey, México. Correo electrónico: catysantosgzz@gmail.com. ORCID: 0000-0002-1362-7975.
} 
ECONOMía TEORÍA y PRÁCTICA [ISSN: 2448-7481] Nueva Época, año 27, número 51, julio-diciembre 2019, Jorge Ibarra Salazar, César González Caloca, Silvia Núñez Gómez, Manuel Ramírez García, Mario Rodríguez Nuncio y Catalina Santos González

consumption of approximately one pack of cigarettes per capita. The regulatory policy towards packaging and labeling has been effective in reducing tobacco demand in Mexico.

Keywords: Tobacco industry regulation, pictorial health warnings, tobacco demand, Mexico.

JEL Classification: I18, L66, L51, C22. 


\section{INTRODUCCIÓN}

Como respuesta coordinada a escala mundial para enfrentar los efectos del tabaquismo sobre la salud, la Organización Mundial de la Salud (OMS) adoptó el Convenio Marco para el Control del Tabaco (СМСT) en mayo de 2003. El Convenio contiene una serie de recomendaciones para regular la industria del tabaco y reducir tanto la demanda como la oferta de este producto (OMS, 2003). Las asociadas a la demanda incluyen la fijación de impuestos, la política de precios e importaciones, la protección a la exposición del humo de tabaco, la reglamentación del contenido del producto, las acciones de divulgación e información, el empaquetado y etiquetado, la educación-concientización, y la publicidad y promoción. Este artículo trata sobre la regulación de etiquetado y empaquetado en la industria de tabaco mexicana.

En mayo de 2004 México ratificó el CMCT y, desde entonces, se han adoptado regulaciones para abatir el tabaquismo. En el contexto del CMCT, y con la intención de reunir en un solo instrumento normativo las diferentes medidas regulatorias, en febrero de 2008 en México se aprobó la Ley General para el Control del Tabaco (LGT) (DOF, 30/04/2008). Las estrategias para reducir la demanda se han implementado de manera escalonada. Reynales et al. (2015) muestran una línea de tiempo de las diferentes regulaciones implementadas en México, en tanto que Reynales et al. (2013) amplían sobre la política pública para el control del tabaco y la Cofemer (2012) sobre la regulación hacia la industria mexicana del tabaco.

Una de las estrategias utilizadas para reducir el tabaquismo es el uso de Advertencias Sanitarias con Pictogramas (ASP). Desde la perspectiva económica, las ASP reducen la asimetría de información y las externalidades negativas en el mercado (Cofemer, 2012). En efecto, los pictogramas son una herramienta de bajo costo que proporciona información sobre los riesgos del tabaquismo tanto a fumadores como a personas que no tienen el hábito de fumar.

La regulación de etiquetado inició en Estados Unidos, cuando en 1966 se requirió la inclusión de texto con una advertencia sanitaria en el costado de las cajetillas de cigarros. En una segunda etapa, durante las décadas de los años ochentas y noventas, los textos de las advertencias aparecieron con diferente tipo de letra y ocupando más espacio en las cajetillas. La tercera etapa inició en 2001 cuando en Canadá aparecieron las Advertencias Sanitarias (ASP) (NCI y WHO, 2016; Monárrez et al., 2014; Hammond, 2009).

Hamilton (1972) fue el primero en analizar los efectos de las ASP sobre el consumo en Estados Unidos. La literatura que estudia estas advertencias es muy amplia. Goel y Nelson (2006), Hammond (2011), Monárrez et al. (2014), Noar et al. (2015), y NCI y WHO (2016), entre otros, revisan la literatura relacionada.

La revisión de la literatura económica sobre la efectividad de la política regulatoria para combatir el tabaquismo de Goel y Nelson (2006), y particularmente 
sobre las advertencias sanitarias, incluye estudios publicados hasta 2003, en los que se analizan advertencias sanitarias textuales en las cajetillas de cigarros. Goel y Nelson (2006) anotan que la evidencia sobre la efectividad de las mismas es mixta. Reconocen que es probable que esto se deba a la dificultad para incorporar las diferencias cualitativas e institucionales de las advertencias sanitarias en los estudios sobre el consumo de tabaco.

Hammond revisa la evidencia sobre la efectividad de las advertencias sanitarias. Incluye, en particular, el efecto diferenciado de las advertencias de texto versus los pictogramas, el impacto en jóvenes versus adultos, el impacto del contenido del mensaje y el impacto en términos del abandono del tabaquismo. Concluye que "La investigación a la fecha enfatiza la importancia del empaque como un medio de comunicación con los fumadores y proporciona evidencia clara de dos precedentes clave en la última década: el uso de pictogramas y el aumento del tamaño de las advertencias en las cajetillas" (Hammond, 2011: 335).

Monárrez et al. (2014) realizan una revisión sistemática de estudios sobre el efecto de los pictogramas en cambios conductuales relacionados con el hábito de fumar. Usaron diferentes criterios para determinar si la probabilidad de que los fumadores abandonen, reduzcan o intenten dejar el hábito de fumar es mayor ante la presencia de las ASP en las cajetillas de cigarros. La revisión de Monárrez et al. (2014) ofrece resultados inconclusos. En su perspectiva, si bien las advertencias sanitarias ilustradas pueden generar algún efecto en la conducta de los fumadores, ésta parece ser modesta en comparación con otras medidas regulatorias hacia la industria del tabaco.

Noar et al. (2015) realizan un meta-análisis de estudios experimentales sobre las ASP. Reportan que los pictogramas son más efectivos que las advertencias sanitarias que sólo contienen texto. Los estudios revisados por Noar et al. (2015) muestran evidencia de que, en comparación con las advertencias textuales, los pictogramas atraen y mantienen mejor la atención, causan reacciones cognitivas y emocionales más fuertes, producen actitudes más negativas hacia el hábito de fumar y son más efectivos para no empezar y dejar de fumar.

La revisión de la literatura sobre las advertencias sanitarias en el reporte de NCI y WHO concluye que "Las advertencias sanitarias grandes en las cajetillas de cigarro son efectivas en tanto que mejoran el conocimiento de los fumadores sobre los efectos del tabaquismo, estimulan su interés por dejar el cigarro y reducen la prevalencia de su consumo. Esas advertencias pueden ser una herramienta especialmente efectiva para informar a niños, jóvenes y personas con poca educación sobre las consecuencias del tabaquismo en la salud" (NCI y WHO, 2016: 303).

Los estudios sobre las ASP en México han evaluado el impacto de la primera ronda de pictogramas en fumadores adultos (Thrasher et al., 2013; Thrasher et al., 
2012a; Thrasher et al., 2012b); reportan sobre el proceso que se siguió para decidir los contenidos textuales y gráficos de la segunda ronda de las ASP (Reid et al., 2013; Thrasher et al., 2013; Regalado et al., 2013; Thrasher et al., 2006) y estiman la reducción en valor de las cajetillas de cigarros al modificar las advertencias sanitarias de texto con imágenes (Thrasher et al., 2007). Estos trabajos concluyen que los fumadores tienen más conocimiento sobre los riesgos de fumar, sobre los componentes tóxicos del tabaco y el número telefónico de ayuda para dejar de fumar. Además, indican que los fumadores están de acuerdo con esta política de empaque y etiquetado.

Si bien estos estudios han notado la efectividad de las ASP en reducir las asimetrías de información y las externalidades negativas en la industria del tabaco, además de que los fumadores asignan un menor valor a los cigarros cuyas cajetillas contienen ASP e infieren que los pictogramas traerían como consecuencia una reducción en la demanda; en ningún estudio se ha estimado la reducción en consumo de tabaco producida por esta medida regulatoria. En tal sentido, la hipótesis que principalmente queremos probar en este artículo es que la política de empaquetado y etiquetado en México ha reducido el consumo per cápita de tabaco. En particular nos interesa obtener una figura numérica estimada sobre el efecto de las ASP sobre el consumo de tabaco en México.

Además, en todos los estudios referidos para México se han empleado datos obtenidos de encuestas aplicadas a personas. En ningún caso se han empleado datos agregados sobre consumo de tabaco para averiguar el efecto de las ASP. Si bien se han utilizado datos en series de tiempo a escala nacional para estimar la elasticidad precio de la demanda por cigarros (Oliviera et al., 2010), no es de nuestro conocimiento un estudio que analice el efecto en México de las ASP sobre la demanda con los datos y métodos empleados en nuestro estudio. La especificación y estimación de los modelos que se aplican en este artículo han sido empleadas para estudiar el efecto de la regulación sobre el consumo de tabaco en otros países (Bardsley y Olekalns, 1999; Nguyen et al., 2012; McLeod, 1986; Pekurinen, 1989; Seldon y Doroodian, 1989; Fujii, 1980).

Este artículo contribuye en los estudios sobre regulación de empaque y etiquetado en México en dos aspectos. En primera instancia proporciona estimaciones de la reducción en el consumo de tabaco como consecuencia de las ASP en las cajetillas de cigarro. En segunda, utiliza datos y métodos que no se han empleado para estudiar el efecto de la regulación de empaque y etiquetado sobre la demanda de tabaco.

Controlando mediante los determinantes de la demanda de tabaco (precio e ingreso per cápita) y por otras regulaciones hacia la industria, el objetivo de este artículo es analizar el impacto sobre la demanda de tabaco en México de las ASP en las cajetillas de cigarrillos. 
Para nuestro análisis estimamos diferentes modelos de demanda de tabaco usando una serie de tiempo trimestral que incluye datos desde el primer trimestre de 1994 hasta el segundo trimestre de 2013. Encontramos evidencia de que la segunda y la tercera rondas de las ASP han reducido en forma significativa la demanda de tabaco en México.

El artículo está organizado de la siguiente manera: en la siguiente sección se presenta la regulación en materia de etiquetado, después la metodología, los resultados y las conclusiones.

\section{REGULACIÓN EN MATERIA DE ETIQUETADO Y EL CONSUMO DE TABACO}

En el último trimestre de 2010 entraron en vigor las especificaciones de las ASP y las leyendas que debían estar impresas en las cajetillas de cigarros (DOF, 24 de diciembre de 2009). Las plantillas fueron modificadas en los meses de septiembre de 2011 (DOF, 9 de mayo de 2011) y 2012 (DOF, 23 de julio de 2012). Si bien nuestro análisis utiliza datos hasta el segundo trimestre de 2013, hacia finales de 2015 se habían aprobado siete rondas de pictogramas. Regalado y Madrazo (2013) presentan el proceso de adopción e implementación de las ASP en México.

La regulación en materia de empaquetado y etiquetado ha sido aplicada en México con base en el capítulo I de la LGT. En el Artículo 18 se menciona que en todo empaquetado y etiquetado externo de productos de tabaco debe aparecer una leyenda y pictograma de advertencia que muestre los efectos nocivos de fumar. Las leyendas y los pictogramas son formulados por la Secretaría de Salud y deben ocupar $30 \%$ de la cara anterior, incluyendo algún pictograma o imagen, $100 \%$ de la cara posterior y $100 \%$ de una de las caras laterales del empaque, los cuales deberán incluir algún mensaje sanitario rotativo, así como un número telefónico de información sobre prevención, cesación y tratamiento de enfermedades o efectos derivados del consumo de tabaco. Estas etiquetas no pueden incluir mensajes que hagan referencia a alguna disposición legal.

El Artículo 19 establece que además de las etiquetas de advertencia, los paquetes de productos de tabaco deberán incluir sus componentes y emisiones. El Artículo 20 prohíbe la promoción de mensajes relacionados con el producto en todo empaquetado y etiquetado externo que resulten engañosos o que pudieran ser mal interpretados. De igual manera se prohíbe emplear términos, elementos descriptivos, marcas de fábrica y signos figurativos que tengan la intención de crear la falsa impresión de que un determinado producto es menos nocivo que otro. Están prohibidas expresiones como bajo contenido de alquitrán, ligeros, ultraligeros o suaves. 
El Artículo 21 establece que en todos los paquetes el empaquetado y etiquetado de productos de tabaco comercializados dentro del país deberá figurar la declaración: Para venta exclusiva en México. Finalmente, el Artículo 22 menciona que las leyendas de advertencia y toda la información textual deberán estar en español en todos los empaques.

El contenido precautorio de las cajetillas ha variado de manera significativa en los últimos diez años. Mientras que en las reformas realizadas a diversas disposiciones de la Ley General de Salud en 2004 (DOF, 19 de enero de 2004) se estipulaba que la cajetilla sólo debía contener leyendas de advertencia, tales como: Fumar es causa de cáncer y enfisema pulmonar; Fumar durante el embarazo aumenta el riesgo de parto prematuro, entre otras. Hoy día se exige la inclusión de pictogramas que muestran explícitamente las consecuencias del consumo de tabaco.

Fue alrededor del último trimestre de 2010 cuando entró en vigor un acuerdo (DOF, 24 de diciembre de 2009) que contenía las especificaciones de los pictogramas y las leyendas que debían estar impresas en las caras de las cajetillas. En este acuerdo se definieron también los plazos en los que se irían introduciendo las distintas plantillas de pictogramas, y se responsabilizaba de la aplicación de las medidas a las empresas productoras e importadoras. Para efectos de ilustración, el cuadro 1 muestra el primer pictograma y los mensajes sanitarios vigentes por un año a partir del 24 de septiembre de 2010.

\section{Cuadro 1. Pictograma y mensaje sanitario del 24 de septiembre de 2010} al 24 de septiembre de 2011

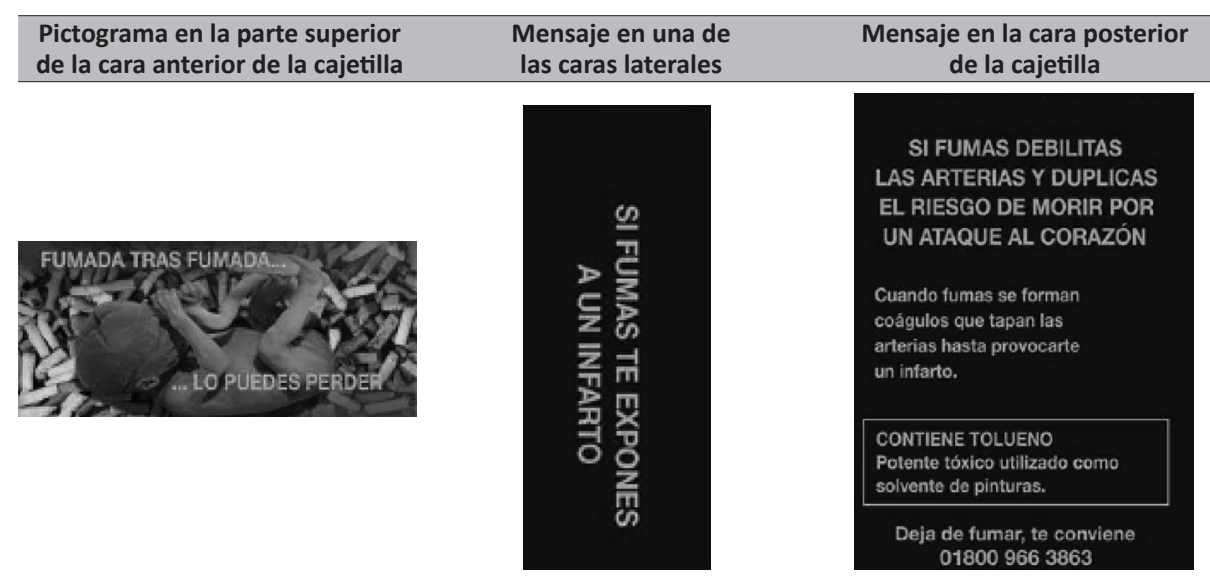

Fuente: DOF, 24 de diciembre de 2009. 
Cuadro 2. Medidas regulatorias del CMCT sobre etiquetado adoptadas por México

\begin{tabular}{|c|c|}
\hline Medidas regulatorias & Adoptado en México \\
\hline Empaque de productos de tabaco no debe de contener anuncios o promoción. & Sí \\
\hline $\begin{array}{l}\text { Programas que requieran que el empaque y etiquetado de cajetillas no promue- } \\
\text { va al producto por medios engañosos. }\end{array}$ & Sí \\
\hline Empaque debe contener etiquetas de advertencia. & Sí \\
\hline Etiquetas de advertencia son aprobadas por la autoridad nacional competente. & Sí \\
\hline Programa que asegure que las etiquetas de advertencia se van rotando. & Sí \\
\hline $\begin{array}{l}\text { Programa que asegure que las etiquetas de advertencia sean claras, visibles y } \\
\text { legibles. }\end{array}$ & Sí \\
\hline $\begin{array}{l}\text { Programa que asegure que las etiquetas de advertencias ocupen no menos de } \\
30 \% \text { del área principal de la cajetilla. }\end{array}$ & Sí \\
\hline $\begin{array}{l}\text { Programa que asegure que las etiquetas de advertencia ocupen } 50 \% \text { o más del } \\
\text { área principal de la cajetilla. }\end{array}$ & Sí \\
\hline Etiquetas de advertencia en forma de pictogramas. & Sí \\
\hline El gobierno tiene los derechos de imagen sobre los pictogramas. & Sí \\
\hline $\begin{array}{l}\text { Cada paquete o etiqueta de tabaco contiene información sobre los componentes } \\
\text { y emisiones del producto. }\end{array}$ & Sí \\
\hline $\begin{array}{l}\text { Se requiere que cualquier información textual de advertencia aparezca en el } \\
\text { idioma del país. }\end{array}$ & Sí \\
\hline
\end{tabular}

Fuente: OMS (2012).

Las plantillas que debían ser impresas en las cajetillas fueron modificadas a partir del 24 de septiembre de 2011, tal como se indica en el Diario Oficial de la Federación del lunes 9 de mayo de 2011. En tal caso se incluyeron como anexo los ocho pictogramas que se debían implementar en los periodos de tiempo estipulados, así como las instrucciones para su impresión, tales como el tipo de letra, el color y su tamaño. Asimismo, se incluye la dimensión del pictograma como porcentaje del área de alguna de las caras de la cajetilla. Posteriormente, para el periodo comprendido del 24 de septiembre de 2012 al 23 de marzo de 2013, se llevó a cabo otra modificación en los pictogramas.

La cuarta ronda de pictogramas (DOF, 30 de noviembre de 2012), que estuvo vigente del 24 de marzo de 2013 al 23 de marzo de 2014, fue distinta a las anteriores. En el conjunto de pictogramas se encuentra escrita en la parte superior de la cara posterior alguna de las enfermedades relacionadas con el tabaquismo, además de expresarse la leyenda: la Secretaría de Salud informa, antes del apartado que describe algunas de las consecuencias del consumo de tabaco. El conjunto de pictogramas vigente del 24 de marzo de 2014 al 23 de septiembre de 2014 (DOF, 3 de enero de 2014) conformó la quinta ronda de ASP impresas en las cajetillas. 
De acuerdo con el reporte de implementación del CMCT de la OMS (2012), México ha adoptado todas las medidas regulatorias en materia de etiquetado. El cuadro 2 resume las medidas regulatorias sobre etiquetado y empaquetado de productos de tabaco contenidas en el Artículo 11 del CMCT, y también indica si esas medidas han sido adoptadas en México.

La figura 1 muestra el comportamiento del consumo per cápita de tabaco, así como la evolución de los precios del tabaco y del piB per cápita. En esa misma figura se marcan las fechas de inicio de las medidas regulatorias consideradas en nuestro estudio. Tal como se aprecia en esa figura, el consumo de tabaco en México se mantuvo en una banda de variación de 110 y 160 cigarros per cápita entre el primer trimestre de 1994 y el tercer trimestre de 2005. A partir de ese trimestre, y después de un repunte en el primer trimestre de 2006, en el que alcanzó 191 cigarros per cápita, el consumo per cápita muestra una tendencia decreciente un tanto irregular: pasó del máximo histórico de 191 en el primer trimestre de 2006 a 80 hacia la mitad de 2013.

Figura 1. México: evolución del consumo per cápita de tabaco, PIB per cápita y el precio del tabaco, primer trimestre 1994-segundo trimestre 2013 (número de cigarros per cápita)

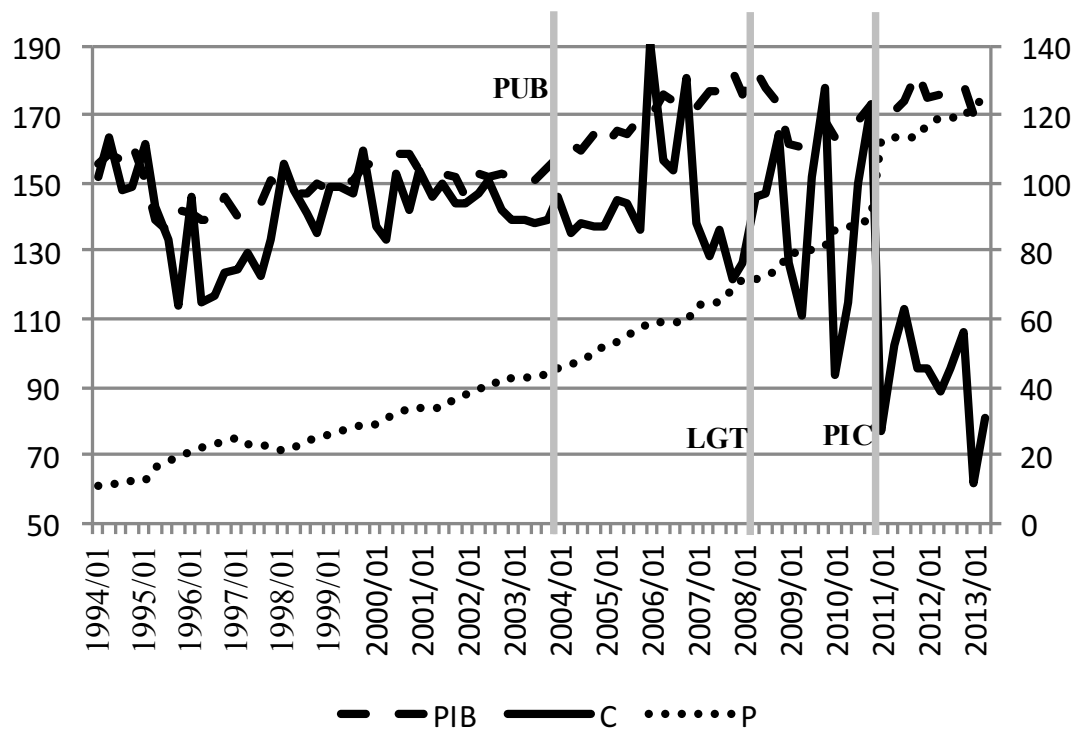

Notas: véase cuadro 3 para descripción de las variables y unidades. PUB marca el trimestre en que entraron en vigor restricciones a la publicidad, LGT marca la fecha en que se promulgó la Ley General para el Control del Tabaco y PIC marca la fecha en que apareció la primera ronda de pictogramas en las cajetillas de cigarros.

Fuente: véase cuadro 3. 
Al contrastar la dinámica del consumo de tabaco con el PIB y el índice de precios de productos de tabaco (figura 1) es interesante distinguir tres relaciones: la caída en el ingreso per cápita de mediados de los noventas coincide con una reducción importante en el consumo; la reducción en el PIB durante 2008 es también consistente con la baja de consumo de tabaco y, aunque desde 2009 el PIB muestra una recuperación paulatina, el consumo de tabaco ha cedido, al parecer como consecuencia del marcado aumento en el precio y la implementación de distintas medidas regulatorias, sobre todo a inicios de 2011.

La figura 2 presenta relaciones parciales entre $\mathrm{C}$ y las variables $\mathrm{P}$ y PIB. El panel de la izquierda muestra una clara relación inversa entre el consumo per cápita y el índice de precios del tabaco, en tanto que el panel de puntos dispersos a la derecha no permite establecer una clara relación entre el PIB y el consumo per cápita.

Figura 2. México: relación del consumo de tabaco con el PIB per cápita y con el precio del tabaco, primer trimestre 1994-segundo trimestre 2013 (número de cigarros per cápita)
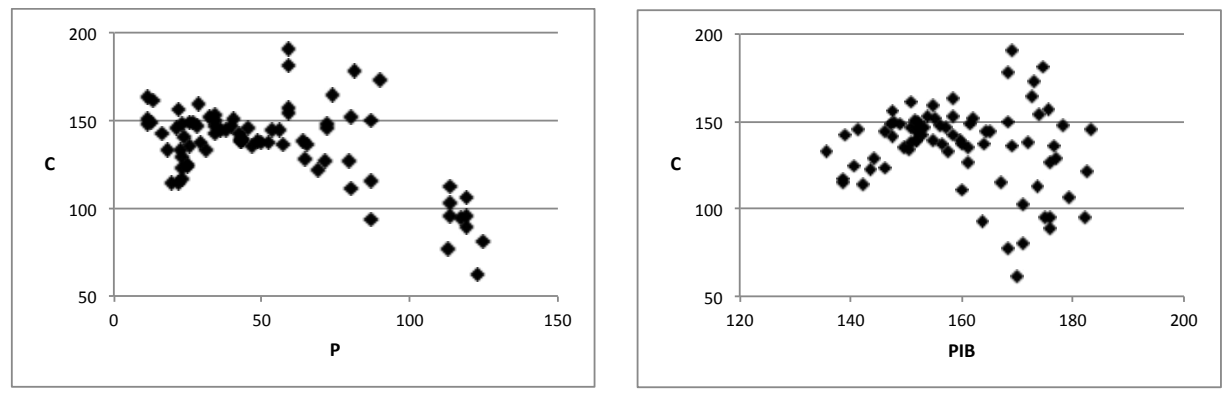

Fuente: véase cuadro 3 para descripción de las variables, unidades y fuentes de información.

\section{METODOLOGÍA}

De acuerdo con lo que indica Cameron (1998), existen estudios sobre la estimación de la demanda de tabaco desde 1933 (Schoenberg, 1933), los cuales se han propagado debido al interés por analizar el impacto de las medidas regulatorias impuestas a la industria del tabaco, sobre todo a raíz de los reportes de salud publicados en Estados Unidos en 1953.

Los estudios sobre demanda de tabaco estiman de forma común el consumo per cápita de cigarrillos tomando como variables independientes el precio del producto, el ingreso de los consumidores y las variables binarias, a fin de reflejar aquellos períodos en los que se implementaron regulaciones hacia la industria 
del tabaco. Algunos autores analizan el contrabando entre estados o países (Baltagi y Levin, 1986; Connelly et al., 2009; Goel y Nelson, 2012), por lo que incluyen como variables explicativas los precios de los estados o países fronterizos. Otros trabajos que estudian los efectos de la regulación han utilizado también índices regulatorios de control del tabaco para cuantificar en forma comparativa el grado de regulación (Baltagi y Levin, 1986; y Nguyen et al., 2012).

Existen tres modelos teóricos que se han utilizado ampliamente en la literatura de demanda de tabaco para motivar los modelos empíricos: el modelo convencional (Pekurinen, 1989; McLeod, 1986; Cox y Smith, 1984); el modelo de adicción miope (Goel y Nelson, 2012; Haden, 1990; Pekurinen, 1989; Seldon y Doroodian, 1989; Baltagi y Levin, 1986), y más recientemente el modelo de adicción racional (Bardsley y Olekalns, 1999; Becker et al., 1994; Sung et al., 1994; Chaloupka, 1991). Nguyen et al. (2012) y Goel y Nelson (2006) explican los tres modelos.

El modelo convencional se basa en la teoría económica del consumidor. La demanda por tabaco en el período $t\left(Q_{t}\right)$ está en función de su precio $\left(P_{t}\right)$, y el ingreso de los individuos $\left(Y_{t}\right)$, así como de las variables relacionadas con medidas regulatorias $\left(R_{t}\right)$ en dicho período:

$$
Q_{t}=f\left(P_{t}, Y_{t}, R_{t}\right)
$$

Este modelo, sin embargo, presenta una limitante importante ya que trata el tabaco como un bien de consumo convencional, ignorando su carácter adictivo. Los modelos miope y racional consideran explícitamente la naturaleza adictiva del tabaco.

El modelo miope fue adaptado del trabajo de Houthakker y Taylor (1966), quienes estimaron un modelo dinámico de demanda que incorpora una ecuación de ajuste para estimar el stock de hábitos de los individuos. Diferente del convencional, el de adicción miope asume que el nivel de consumo en el período $t$ depende del consumo en el período anterior $(t-1)$ :

$$
Q_{t}=f\left(P_{t}, Y_{t}, R_{t}, Q_{t-1}\right)
$$

La idea de la persistencia de hábitos se puede motivar también a través del modelo de ajuste parcial, relacionando la demanda de tabaco deseada $\left(Q_{t}^{*}\right)$ y la observada $\left(Q_{t}\right)$. Para efectos de ilustración, si la demanda deseada es función de las variables independientes y adopta la forma funcional lineal:

$$
Q_{t}^{*}=\beta_{0}+\beta_{1} P_{t}+\beta_{2} Y_{t}+\beta_{3} R_{t}
$$


Luego se relaciona la demanda deseada con la observada de la siguiente forma:

$$
Q_{t}-Q_{t-1}=\delta\left(Q_{t}^{*}-Q_{t-1}\right)
$$

En este caso: $0<\delta \leq 1$ es el coeficiente de ajuste entre la diferencia de la demanda deseada y la observada en relación con la demanda del período anterior. Combinando (3) con (4) se obtiene la expresión que relaciona la demanda observada en función de las variables independientes contemporáneas y la demanda rezagada un período:

$$
Q_{t}=\delta \beta_{0}+\delta \beta_{1} P_{t}+\delta \beta_{2} Y_{t}+\delta \beta_{3} R_{t}+(1-\delta) Q_{t-1}
$$

Este modelo supone que las decisiones de consumo de bienes adictivos en el presente se realizan tomando en cuenta nociones como la tolerancia, ya que un determinado nivel de consumo genera menor satisfacción conforme sea mayor el consumo pasado. De esta manera supone una relación directa entre el consumo pasado y el consumo presente. El consumidor aprende también la reacción que ha tenido hacia el bien adictivo en el pasado, por lo que éste podría usarse, por ejemplo, como método para aliviar la tensión o el estrés. No obstante, el modelo miope no toma en cuenta que los consumidores también pueden valorar los efectos en su salud como consecuencia del consumo de tabaco.

Hacia finales de los años ochentas, y con base en los trabajos de Stigler y Becker (1977), Becker y Murphy (1988) y Becker et al. (1994), desarrollaron un modelo teórico para explicar la demanda de bienes adictivos. Ellos propusieron una teoría de adicción racional en la que los consumidores maximizan su utilidad al decidir cuánto consumir de un bien adictivo, tomando en cuenta no sólo la adicción del consumidor, sino también las consecuencias futuras de sus acciones.

La interacción entre el consumo en distintos períodos sucede a través de la utilidad marginal de consumir el bien adictivo. El aumento del consumo del bien adictivo en el período pasado ocasionará que la utilidad marginal de consumirlo en el presente sea mayor que el valor presente del daño marginal que habría al consumirlo en el futuro (Becker et al., 1994).

Este modelo aplica no sólo en bienes adictivos como el tabaco y el alcohol, sino que puede ser utilizado también para comportamientos adictivos como el trabajo, la manera de vivir, religión, etcétera. Se dice que un bien es potencialmente adictivo si un aumento en el consumo pasado ocasiona que el consumo presente aumente, mientras que un aumento esperado de precios en el futuro 
disminuirá el consumo presente. Por eso se dice que los niveles de consumo en diferentes momentos en el tiempo son bienes complementarios.

En este modelo la demanda de tabaco en el período actual depende tanto de la demanda del período anterior como de la del período posterior $(t+1)$ :

$$
Q_{t}=f\left(P_{t}, Y_{t}, R_{t}, Q_{t-1}, Q_{t+1}\right)
$$

En este caso también se espera una relación directa entre el consumo pasado y el presente, dado que el primero incentiva al segundo al aumentar la utilidad marginal en una magnitud mayor que el valor presente del daño futuro esperado por el consumo del bien adictivo.

Para la estimación de los modelos en nuestro estudio, usamos una serie de 78 observaciones trimestrales desde el primer trimestre de 1994 hasta el segundo trimestre de 2013. El cuadro 3 describe las variables utilizadas y sus fuentes.

Para aproximar el comportamiento de la demanda nacional utilizamos el consumo de cigarrillos per cápita (C). El consumo se midió con la suma de la producción nacional e importaciones netas de tabaco. Este enfoque ha sido empleado por Guidon y Boisclair (2003) y Ahsan et al. (2014). En su estudio de la demanda de tabaco en México, Olivera et al. (2010) y Waters et al. (2010) cuantifican el consumo de la misma forma que lo hacemos en el presente trabajo.

Las variables explicativas de la demanda de tabaco consisten en el precio del tabaco y el Producto Interno Bruto (PIB) trimestral per cápita. Las regulaciones consideradas como controles en los modelos son: la reforma de 2004 a la Ley General de Salud (DOF, 19 de enero de 2004), que incorporó los artículos 308 bis y 309 bis prohibiendo la publicidad de productos de tabaco en radio y televisión, así como la aprobación de la LGT. Con esta segunda variable incorporamos los instrumentos regulatorios que se han implementado en México para reducir la demanda, distintos de las ASP. La primera variable la denotamos como PUB y la segunda como LGT.

Las medidas regulatorias de interés son las tres rondas de ASP (PIC1, PIC2 y PIC3). Como es común en los estudios que utilizan datos agregados en series de tiempo, definimos variables binarias para incorporar el efecto de la regulación de etiquetado (Baltagi y Levin, 1986, 1992; Goel y Morey, 1995; Hamilton, 1972). De acuerdo con nuestra hipótesis, esperamos que los parámetros asociados a estas variables sean estadísticamente significativos.

Estimamos los tres modelos teóricos expuestos en dos formas funcionales: lineal y logarítmica. La estrategia de estimación fue la siguiente. En primera instancia analizamos las propiedades de las series de tiempo de las variables usadas. Probamos si las series eran estacionarias, para corroborar que no tuvieran raíz unitaria. Realizamos la prueba de Dickey-Fuller aumentada (ADF) para analizar 
la estacionalidad de las variables y así establecer su orden de integración. Para determinar la presencia de cointegración utilizamos la metodología de Engle y Granger (1987).

Después de estimar cada ecuación por mínimos cuadrados ordinarios, se probó si existía autocorrelación y heteroscedasticidad y se determinó, en su caso, la severidad de la multicolinealidad. Para probar la existencia de heteroscedasticidad y autocorrelación en los errores se usaron las pruebas de Breush-Pagan y Breusch-Godfrey, ya que los datos son una serie de tiempo; en caso de que los modelos presentaran ambos problemas, se optó por corregir primero la autocorrelación realizando la transformación de Prais-Winsten.

En cuanto a la multicolinealidad, dado que se partió de especificaciones de modelos teóricos particulares, no se tomaron acciones para aminorarla. Transformar o eliminar variables independientes habría modificado la esencia misma del modelo.

De ser el caso, una vez corregidos los problemas de autocorrelación y heteroscedasticidad, se utilizaron distintas combinaciones de variables instrumentales para determinar estadísticamente si existía el problema de endogeneidad. En efecto, los estudios relacionados sugieren que al incluir un rezago o un adelanto en los modelos de demanda de tabaco se puede generar un problema de endogeneidad (Nguyen et al., 2012). Esto se debe a que el rezago y/o el adelanto de la variable dependiente pueden estar correlacionados con el error o sus rezagos.

Otro argumento para la existencia de endogeneidad en la estimación de funciones de demanda, en el caso de modelos de equilibrio parcial, es el problema de identificación, ya que precios y cantidades se determinan de forma simultánea (Wilkins et al., 2001). Sin embargo, la naturaleza de los mercados puede ayudar para amainar esta sospecha. En el caso particular del mercado de productos de tabaco, existen motivos para creer que el precio puede comportarse de manera exógena. Por un lado, diversos estudios empíricos han caracterizado el mercado de productos de tabaco como un oligopolio (Bhuyan y López, 1997; Appelbaum, 1982; Barnett et al., 1995; Delipalla y O'Donnell, 2001). Por el otro, dado que la fijación de impuestos y el control de precios ha sido una herramienta aplicada en el mercado de tabaco, el precio de esos productos está influido por estas medidas para contener el tabaquismo.

En atención a los estudios de Wilkins et al. (2003) y Nguyen et al. (2012) utilizamos como variables instrumentales el impuesto al tabaco, así como rezagos $\mathrm{y}$ adelantos de sus precios.

En nuestro caso, se usó la prueba de especificación de Hausman (1978) para detectar la endogeneidad en los modelos de adicción miope y racional. Al encontrar evidencia de endogeneidad, el modelo fue estimado por mínimos cuadrados de dos etapas con variables instrumentales. Se presentaron las pruebas 
para diferentes combinaciones de instrumentos. En los modelos de adicción miope se utilizan dos rezagos y dos adelantos del precio, un rezago y un adelanto del precio, dos rezagos y dos adelantos del precio. En los modelos de adicción racional se utilizaron los mismos instrumentos y se añadió el IEPS. El número de instrumentos en cada caso fue para tener un mayor número de instrumentos que de variables potencialmente endógenas, y de esta manera probar estadísticamente su validez.

En la prueba de Hausman la hipótesis nula es que las variables del rezago y el adelanto de la variable dependiente son exógenas. Al rechazar esta hipótesis existe evidencia de endogeneidad. La prueba de validez de instrumentos nos ayuda a determinar si el modelo está sobreidentificado, en cuyo caso los instrumentos serían válidos. Al rechazar la hipótesis nula de sobreidenificación habría evidencia de que los instrumentos pudieran no ser válidos.

Cuadro 3. Descripción de las variables y fuentes de información

\begin{tabular}{lll}
\hline Variable $\quad$ Descripción & Unidades & Fuente \\
\hline Variable Dependiente &
\end{tabular}

\begin{tabular}{|c|c|c|c|}
\hline $\mathrm{C}$ & $\begin{array}{l}\text { Producción nacional de } \\
\text { tabaco + importaciones } \\
\text { netas de tabaco. }{ }^{\text {a }}\end{array}$ & Cigarrillos per cápita. & $\begin{array}{l}\text { Olivera et al. (2010), Secreta- } \\
\text { ría de Economía, }{ }^{c} \text { INEGI. }{ }^{d}\end{array}$ \\
\hline \multicolumn{4}{|c|}{ Variables Independientes } \\
\hline$P$ & $\begin{array}{l}\text { Precio del tabaco } \\
\text { aproximado con el subíndice } \\
\text { de precios del tabaco. }{ }^{\mathrm{b}}\end{array}$ & $\begin{array}{l}\text { Índice de precios con base } \\
\text { en } 2010 .\end{array}$ & INEGI www.inegi.gob.mx \\
\hline PIB & $\begin{array}{l}\text { Producto Interno Bruto } \\
\text { trimestral per cápita } \\
\text { (población mayor de } 15 \\
\text { años). }\end{array}$ & Miles de pesos de 2010. & $\begin{array}{l}\text { Banco de información } \\
\text { económica del INEGI y } \\
\text { Conapo. }\end{array}$ \\
\hline PIC1 & $\begin{array}{l}\text { Variable binarias de la } \\
\text { primera ronda de } \\
\text { pictogramas. }\end{array}$ & $\begin{array}{l}\text { Toma el valor de uno ( } 1 \text { ) del } \\
\text { cuarto trimestre de } 2010 \text { al tercer } \\
\text { trimestre de } 2011 \text { y cero (0) en } \\
\text { cualquier otro caso. }\end{array}$ & $\begin{array}{l}\text { DOF, } 24 \text { de diciembre de } \\
2009 .\end{array}$ \\
\hline PIC2 & $\begin{array}{l}\text { Variable binaria de la } \\
\text { segunda ronda de } \\
\text { pictogramas. }\end{array}$ & $\begin{array}{l}\text { Toma el valor de uno ( } 1 \text { ) del } \\
\text { cuarto trimestre de } 2011 \text { al tercer } \\
\text { trimestre de } 2012 \text { y cero (0) en } \\
\text { cualquier otro caso. }\end{array}$ & DOF, 9 de mayo de 2011. \\
\hline
\end{tabular}


ECONOMía TEORÍA y PRÁCTICA [ISSN: 2448-7481] - Nueva Época, año 27, número 51, julio-diciembre 2019, Jorge Ibarra Salazar, César González Caloca, Silvia Núñez Gómez, Manuel Ramírez García, Mario Rodríguez Nuncio y Catalina Santos González

Cuadro 3. Continuación.

\begin{tabular}{|c|c|c|c|}
\hline Variable & Descripción & Unidades & Fuente \\
\hline \multicolumn{4}{|c|}{ Variables Independientes } \\
\hline PIC3 & $\begin{array}{l}\text { Variable binaria de la } \\
\text { tercera ronda de } \\
\text { pictogramas. }\end{array}$ & $\begin{array}{l}\text { Toma el valor de uno }(1) \text { del } \\
\text { cuarto trimestre de } 2012 \text { al primer } \\
\text { trimestre de } 2013 \text { y cero }(0) \text { en } \\
\text { cualquier otro caso. }\end{array}$ & DOF, 23 de julio de 2012. \\
\hline LGT & $\begin{array}{l}\text { Variable binaria para } \\
\text { reconocer la aprobación } \\
\text { de la LGT. }\end{array}$ & $\begin{array}{l}\text { Toma el valor de uno ( } 1 \text { ) desde el } \\
\text { primer trimestre de } 2008 \text { y cero } \\
\text { en cualquier otro caso. }\end{array}$ & DOF, 30 de abril de 2008 . \\
\hline PUB & $\begin{array}{l}\text { Variable para reconocer las } \\
\text { restricciones a la publicidad } \\
\text { de productos de tabaco. }\end{array}$ & $\begin{array}{l}\text { Toma el valor de uno (1) a partir } \\
\text { del primer trimestre de } 2004 \text { y } \\
\text { cero (0) en cualquier otro caso. }\end{array}$ & DOF, 19 de enero de 2004. \\
\hline
\end{tabular}

Notas: a La información sobre la producción nacional para los años de 1994 a 2004 sólo toma en cuenta la información de los cigarrillos con filtro, debido a que de 2001 a 2005 no se publicó información sobre los cigarrillos sin filtro. Para los años de 2005 a 2013 la variable comprende los cigarros de todo tipo. Debido a que la información sobre la producción nacional viene expresada en cajetillas, mientras que la relacionada con importaciones netas viene expresada en kilogramos, fue necesario aplicar un factor de conversión para uniformar las unidades de medición. Una cajetilla equivale a 20 cigarros y

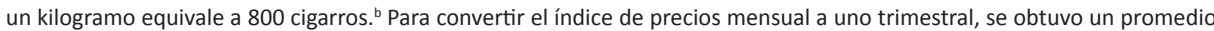
entre los tres valores de los meses que corresponden a cada trimestre. ${ }^{`}$ Sistema de Información Arancelaria vía internet, en: http://200.77.231.38/siavi4/fraccion.php. ${ }^{d}$ Encuesta Mensual de la Industria Manufacturera, en: www.inegi.gob.mx

Fuente: De 2005 a 2006 la información proviene de la Encuesta Industrial Mensual CMAP; para el período de 2007 al segundo trimestre de 2013 los datos provienen de la EMIM. La información de las importaciones netas pertenece al capítulo 24 del SIAVI, Tabaco y sucedáneos del tabaco elaborado. Este capítulo sólo toma en cuenta la fracción 2402.20.01, Cigarrillos que contengan tabaco.

\section{RESULTADOS}

Con respecto a las propiedades de las series de tiempo, tanto en la versión en niveles como en la logarítmica, encontramos que las variables empleadas en este artículo, sin incluir las binarias, tienen algún tipo de tendencia. En el cuadro 4 se presentan las pruebas de Dickey-Fuller utilizadas para probar la existencia de raíz unitaria en las series. Empleamos un rezago de la serie, así como especificaciones con y sin intercepto, ya que esta prueba es sensible tanto a la especificación utilizada como al número de datos.

En todos los casos, los estadísticos de las pruebas Dickey-Fuller no permiten rechazar la hipótesis nula de que las series tengan raíz unitaria, tanto en niveles como en logaritmos. Por su parte, las pruebas realizadas en la primera diferencia 
de cada una de las series rechazan la hipótesis nula de no-estacionariedad. Por lo tanto, se concluye que las series en niveles son integradas de orden uno, mientras que las series en primera diferencia son integradas de orden cero.

El estadístico de prueba Granger-Engle de cointegración para el modelo en niveles fue de -5.77, y para el modelo en logaritmos de -6.18. En ambos casos el valor del estadístico de prueba fue menor a los valores teóricos, por lo que los residuales de las regresiones son integradas de orden cero, lo que implica que las variables están cointegradas. Al rechazar la hipótesis nula de no-estacionariedad, los residuales son de orden cero y las variables están cointegradas. Así, aunque las variables son integradas de orden uno, es adecuado estimar los modelos lineal y logarítmico en niveles.

En el cuadro 5 se proporcionan a manera de referencia los resultados de las estimaciones por mínimos cuadrados ordinarios. Los estadísticos de prueba para heteroscedasticidad (Breusch-Pagan) y autocorrelación (Breusch-Godfrey) se muestran en los penúltimos renglones de este cuadro. En los modelos lineales no fue posible rechazar las hipótesis de homoscedasticidad y no-autocorrelación, mientras que aquéllos estimados en logaritmos naturales presentaron heteroscedasticidad. Además, el modelo de adicción miope, estimado en logaritmos naturales, también presenta autocorrelación. De esta forma, y para efectos de análisis, se tomó la estimación por mínimos cuadrados ordinarios del modelo convencional; en tanto que, ante la presencia de heteroscedasticidad, los modelos en logaritmos naturales fueron estimados por mínimos cuadrados ordinarios con errores robustos; finalmente, al modelo de adicción miope se le aplicó la transformación Prais-Winsten (cuadro 7).

Como se mencionó en la sección de metodología, en los modelos de adicción miope y racional la inclusión de rezagos y adelantos del consumo de tabaco puede crear el problema de endogeneidad. De forma como se sugiere en trabajos relacionados, las variables instrumentales que se han utilizado son comunes en la estimación de los modelos de adicción miope y racional (Wilkins et al., 2003; Nguyen et al., 2012). Además, es interesante notar que, de acuerdo con la regla práctica de correlación parcial entre las variables que se sospechan endógenas y las variables instrumentales, el consumo de tabaco rezagado tiene una correlación de -0.5 con el primer rezago del precio, una de -0.49 con el segundo y una correlación de 0.42 con el adelanto del precio. De igual modo, el consumo adelantado de tabaco muestra una correlación de -0.5 y -0.54 con el rezago y adelanto del precio, respectivamente.

Los estadísticos de prueba para determinar si los modelos de adicción miope y de adicción racional presentan endogeneidad se muestran en el cuadro 6 . En ese mismo cuadro se incluye el estadístico de prueba para validar los instrumentos. La especificación en niveles del modelo de adicción miope, donde las 
variables instrumentales son dos rezagos del precio del tabaco, presenta endogeneidad.

Con respecto al modelo de adicción racional podemos apreciar en el cuadro 6 que la especificación en niveles con un rezago y un adelanto del precio del tabaco, y con el IEPS, muestra evidencia de endogeneidad y de que los instrumentos son adecuados.

El cuadro 7 contiene los resultados de los modelos estimados de acuerdo con el proceso de calibración descrito en la metodología. Es interesante notar que los resultados de los diferentes modelos y especificaciones son consistentes en cuanto a la relación que guardan las variables independientes P y PIB con la demanda de tabaco. En todos los casos el precio guarda una relación inversa, y el ingreso per cápita una relación directa con la demanda de tabaco. Las estimaciones de los distintos modelos teóricos en la especificación lineal muestran, además, que tanto el parámetro del precio como aquél de la variable ingreso son estadísticamente diferentes de cero.

Para discriminar entre los modelos estimados en la especificación en niveles, notemos, en los modelos de adicción miope y racional, que los parámetros del consumo rezagado no son significativos estadísticamente. Esto indica que la hipótesis de adicción en esos modelos no se cumple, ya que el consumo actual no depende del rezagado. De acuerdo con la estimación de la especificación en niveles del modelo convencional, si el índice de precios del tabaco aumenta diez puntos, entonces la demanda se reduciría en 7.5 cigarros per cápita. Asimismo, un aumento en el ingreso per cápita de mil pesos aumentará también el consumo per cápita de tabaco en 1.16 cigarrillos.

La especificación logarítmica de los tres modelos estimados muestra que los parámetros de las variables precio e ingreso per cápita son estadísticamente significativos, sólo con excepción del parámetro del precio en el modelo de adicción miope. En las especificaciones logarítmicas los coeficientes de las variables continuas proporcionan un estimado de las elasticidades de la demanda de tabaco. En particular, de acuerdo con el modelo convencional, la elasticidad precio de la demanda de tabaco es de -0.1030 , y la elasticidad de la demanda con respecto al ingreso estimada es de 1.2848 .

Toda vez que el parámetro de la variable rezagada en la especificación logarítmica del modelo de adicción miope es estadísticamente significativo, habría evidencia para validar los postulados de este modelo. El parámetro estimado de la variable rezagada para la demanda de tabaco permite obtener el factor que representa la proporción del ajuste total en la demanda que se materializa en la demanda de tabaco observada: $1-0.3236=0.6764$. De esta forma, la elasticidad precio de la demanda en el largo plazo sería el parámetro de la variable precio 
$(-0.068)$, mientras que la de corto plazo es de -0.046 , que se obtiene multiplicando el factor de ajuste por la elasticidad de largo plazo.

Estas elasticidades contrastan con las estimadas por Olivera et al. (2010) para el período 1994-2005. Al usar mínimos cuadrados ordinarios estiman que la elasticidad precio de la demanda es de -0.1414 , mientras que en el largo plazo su estimado es de -0.25 al aplicar mínimos cuadrados ordinarios dinámicos. Las diferencias se pueden explicar por la desigualdad en el período de tiempo considerado, y por los distintos métodos de estimación.

De acuerdo con los resultados del mismo modelo, nuestro estimado de la elasticidad ingreso de largo plazo es de 1.03, mientras que para el corto plazo es de 0.697 .

El coeficiente de la variable que indica la aprobación de la LGT no es estadísticamente significativo en ninguno de los modelos estimados. En relación con la variable PUB, los resultados muestran una relación negativa en las especificaciones logarítmicas, aunque es estadísticamente significativa sólo en el modelo de adicción racional.

El interés central de este trabajo es el efecto de la regulación de empaque y etiquetado a través de las ASP. Las variables asociadas a las tres rondas de ASP analizadas en este estudio muestran un coeficiente negativo en todos los modelos estimados, que es estadísticamente significativo para la segunda y la tercera rondas en los tres modelos de las especificaciones en niveles y logarítmica.

De acuerdo con los parámetros estimados de la especificación en niveles del modelo convencional, la segunda ronda de las ASP redujo el consumo en 22 cigarrillos per cápita, en tanto que la tercera ronda en 26. Así, según la especificación logarítmica, el parámetro estimado para PIC2 es de -0.3113 . La exponencial de este parámetro (0.7325) representa el cociente entre la demanda cuando la segunda ronda de pictogramas estuvo vigente y la demanda de tabaco cuando dicha ronda no estuvo vigente. Con base en esto, la demanda de tabaco se habría reducido $26.8 \%$ con la segunda ronda de pictogramas. Con este mismo modelo, el parámetro estimado para la tercera ronda de pictogramas (-0.4617) implicaría que la reducción en la demanda fue alrededor de un $37 \%$.

El modelo de adicción miope, en su especificación logarítmica, permite calcular y comparar la reducción en la demanda en el corto y largo plazos. El parámetro estimado para la segunda ronda de pictogramas $(-0.2266)$ indica una reducción en la demanda de 20.3 \% en el largo plazo; en tanto que al usar de nuevo el factor de ajuste estimado en este modelo (0.6764), resulta que el efecto de corto plazo fue de $14.2 \%$. Para la tercera ronda de pictogramas el parámetro estimado (-0.4215) implica una reducción en la demanda de tabaco de $24.8 \%$ en el corto plazo y de $34.4 \%$ en el largo plazo. 
ECONOMía TEORÍA y PRÁCTICA [ISSN: 2448-7481] - Nueva Época, año 27, número 51, julio-diciembre 2019, Jorge Ibarra Salazar, César González Caloca, Silvia Núñez Gómez, Manuel Ramírez García, Mario Rodríguez Nuncio

y Catalina Santos González

Cuadro 4. Estadísticos de prueba para estacionariedad

\begin{tabular}{|c|c|c|c|c|c|}
\hline & \multicolumn{3}{|c|}{ Dickey-Fuller sin intercepto } & \multicolumn{2}{|c|}{ Dickey-Fuller con intercepto } \\
\hline & $\mathbf{N}$ & $\begin{array}{c}\text { D-F } \\
\text { (calculado) }\end{array}$ & $\begin{array}{l}\text { Orden de } \\
\text { integración }\end{array}$ & $\begin{array}{c}\text { D-F } \\
\text { (calculado) }\end{array}$ & $\begin{array}{c}\text { Orden de } \\
\text { integración }\end{array}$ \\
\hline PIB & 78 & 0.5 & $\mathrm{I}(1)$ & -0.82 & $\mathrm{I}(1)$ \\
\hline C & 78 & -0.8 & $\mathrm{I}(1)$ & -4.08 & $\mathrm{I}(1)$ \\
\hline$P$ & 78 & 3.9 & $\mathrm{I}(1)$ & 1.48 & $\mathrm{I}(1)$ \\
\hline $\ln P I B$ & 78 & 0.5 & $\mathrm{I}(1)$ & -0.89 & $\mathrm{I}(1)$ \\
\hline $\ln C$ & 78 & -0.4 & $\mathrm{I}(1)$ & -3.82 & $\mathrm{I}(1)$ \\
\hline $\ln P$ & 78 & 3.3 & $\mathrm{I}(1)$ & -1.75 & $\mathrm{I}(1)$ \\
\hline$\Delta(\mathrm{PIB})$ & 77 & -5.6 & $\mathrm{I}(0)$ & -5.56 & $\mathrm{I}(0)$ \\
\hline$\Delta(\mathrm{C})$ & 77 & -12.0 & $\mathrm{I}(0)$ & -11.94 & $\mathrm{I}(0)$ \\
\hline$\Delta(\mathrm{P})$ & 77 & -4.9 & $I(0)$ & -6.64 & $I(0)$ \\
\hline$\Delta(\ln \mathrm{PIB})$ & 77 & -5.7 & $I(0)$ & -5.69 & $I(0)$ \\
\hline$\Delta(\ln C)$ & 77 & -3.6 & $I(0)$ & -12.11 & $I(0)$ \\
\hline \multirow[t]{2}{*}{$\Delta(\ln P)$} & 77 & -12.1 & $I(0)$ & -5.1 & $I(0)$ \\
\hline & & $1 \%$ & $5 \%$ & $1 \%$ & $5 \%$ \\
\hline Valores críticos D-F & & -2.6 & -1.95 & -3.51 & -2.89 \\
\hline
\end{tabular}

Notas. Véase cuadro 3 para los nombres de las variables. No se incluyó tendencia. D-F se refiere a Dickey-Fuller. N es el número de observaciones. Los valores críticos D-F se obtuvieron de Enders (2010). $\Delta$ se refiere a la primera diferencia. 


\section{Cuadro 5. Resultados de las estimaciones por mínimos cuadrados ordinarios}

Modelo convencional:

Lineal: $C=\beta_{0}+\beta_{1}$ P $+\beta_{2}$ PIB $+\beta_{3}$ LGT $+\beta_{4}$ PUB $+\beta_{5}$ PIC $1+\beta_{6}$ PIC $2+\beta_{7}$ PIC 3

Logaritmos: In $C=\beta_{0}+\beta_{1} \operatorname{InP}+\beta_{2} \operatorname{InPIB}+\beta_{3}$ LGT $+\beta_{4}$ PUB $+\beta_{5}$ PIC1 $+\beta_{6}$ PIC2 $+\beta_{7}$ PIC3

Modelo de adicción miope:

Lineal: $C=\beta_{0}+\beta_{1}$ P $+\beta_{2}$ PIB $+\beta_{3}$ LGT $+\beta_{4}$ PUB $+\beta_{5}$ PIC $1+\beta_{6}$ PIC $2+\beta_{7}$ PIC $3+\beta_{8} C_{t-1}$

Logaritmos: $\ln C=\beta_{0}+\beta_{1} \ln \mathrm{P}+\beta_{2} \ln$ PIB $+\beta_{3}$ LGT $+\beta_{4}$ PUB $+\beta_{5}$ PIC1 $+\beta_{6}$ PIC2 $+\beta_{7}$ PIC $3+\beta_{8} \ln C_{t-1}$

Modelo de adicción racional:

Lineal: $\mathrm{C}=\beta_{0}+\beta_{1} \mathrm{P}+\beta_{2}$ PIB $+\beta_{3} \mathrm{LGT}+\beta_{4}$ PUB $+\beta_{5}$ PIC1 $+\beta_{6}$ PIC $2+\beta_{7}$ PIC $3+\beta_{8} C_{t-1}+\beta_{9} C_{t+1}$ Logaritmos: $\ln C=\beta_{0}+\beta_{1} \ln$ P $+\beta_{2} \ln$ PIB $+\beta_{3}$ LGT $+\beta_{4}$ PUB $+\beta_{5}$ PIC1 $+\beta_{6}$ PIC $2+\beta_{7}$ PIC $3+\beta_{8} \ln C_{t-1}+\beta_{9}$ $\ln \mathrm{C}_{\mathrm{t}+1}$

\begin{tabular}{|c|c|c|c|c|c|c|}
\hline \multirow[b]{2}{*}{ Variable } & \multicolumn{3}{|c|}{ Lineal } & \multicolumn{3}{|c|}{ Logaritmos } \\
\hline & Convencional & Miope & Racional & Convencional & Miope & Racional \\
\hline$P$ & $\begin{array}{l}-0.7515^{* * *} \\
(0.2055)\end{array}$ & $\begin{array}{l}-0.7034^{* * *} \\
(0.2175)\end{array}$ & $\begin{array}{l}-0.5779 * * \\
(0.2270)\end{array}$ & & & \\
\hline PIB & $\begin{array}{l}1.1609 * * * \\
(0.2836)\end{array}$ & $\begin{array}{l}1.1099 * * * \\
(0.3253)\end{array}$ & $\begin{array}{l}1.1053^{* * *} \\
(0.3927)\end{array}$ & & & \\
\hline $\ln P$ & & & & $\begin{array}{l}-0.1030^{* *} \\
(0.0042)\end{array}$ & $\begin{array}{c}-0.0680 \\
(0.0503)\end{array}$ & $\begin{array}{l}-0.0791^{* *} \\
(0.0368)\end{array}$ \\
\hline $\ln P I B$ & & & & $\begin{array}{l}1.2848^{* * *} \\
(0.3265)\end{array}$ & $\begin{array}{l}1.0309 * * * \\
(0.3609)\end{array}$ & $\begin{array}{l}1.3675^{* * *} \\
(0.4915)\end{array}$ \\
\hline LGT & $\begin{array}{c}6.8282 \\
(10.1767)\end{array}$ & $\begin{array}{c}7.8191 \\
(10.2152)\end{array}$ & $\begin{array}{c}5.5857 \\
(10.3414)\end{array}$ & $\begin{array}{c}-0.0802 \\
(0.0658)\end{array}$ & $\begin{array}{c}-0.0361 \\
(0.0496)\end{array}$ & $\begin{array}{l}-0.0500 \\
(0.0702)\end{array}$ \\
\hline PUB & $\begin{array}{c}3.8235 \\
(6.8322)\end{array}$ & $\begin{array}{c}2.5729 \\
(6.4316)\end{array}$ & $\begin{array}{c}-0.7076 \\
(6.3858)\end{array}$ & $\begin{array}{l}-0.0478 \\
(0.0452)\end{array}$ & $\begin{array}{c}-0.0561 \\
(0.0618)\end{array}$ & $\begin{array}{c}-0.0774 * \\
(0.0444)\end{array}$ \\
\hline PIC1 & $\begin{array}{c}-2.3961 \\
(13.6722)\end{array}$ & $\begin{array}{c}-3.2923 \\
(13.3739)\end{array}$ & $\begin{array}{c}-7.2815 \\
(15.3189)\end{array}$ & $\begin{array}{c}-0.1181 \\
(0.1275)\end{array}$ & $\begin{array}{l}-0.1328^{* *} \\
(0.0630)\end{array}$ & $\begin{array}{l}-0.1796 \\
(0.1356)\end{array}$ \\
\hline PIC2 & $\begin{array}{c}-22.1216^{* * *} \\
(8.2849)\end{array}$ & $\begin{array}{c}-19.4631^{* * *} \\
(7.3203)\end{array}$ & $\begin{array}{l}-26.0838^{* *} \\
(10.5431)\end{array}$ & $\begin{array}{l}-0.3113 * * * \\
(0.0268)\end{array}$ & $\begin{array}{l}-0.2266^{* * *} \\
(0.0746)\end{array}$ & $\begin{array}{l}-0.3621^{* * *} \\
(0.1005)\end{array}$ \\
\hline PIC3 & $\begin{array}{l}-26.4450^{*} \\
(15.2306)\end{array}$ & $\begin{array}{l}-24.6706 \\
(15.3060)\end{array}$ & $\begin{array}{l}-32.5933 \\
(20.2098)\end{array}$ & $\begin{array}{l}-0.4617^{* *} \\
(0.1889)\end{array}$ & $\begin{array}{l}-0.4215^{* * *} \\
(0.1019)\end{array}$ & $\begin{array}{l}-0.5545^{* *} \\
(0.2328)\end{array}$ \\
\hline $\mathrm{C}_{\mathrm{t}-1}$ & & $\begin{array}{c}0.1219 \\
(0.1455)\end{array}$ & $\begin{array}{c}0.0734 \\
(0.1453)\end{array}$ & & & \\
\hline
\end{tabular}


ECONOMía TEORÍA y PRÁCTICA [ISSN: 2448-7481] - Nueva Época, año 27, número 51, julio-diciembre 2019, Jorge Ibarra Salazar, César González Caloca, Silvia Núñez Gómez, Manuel Ramírez García, Mario Rodríguez Nuncio

Cuadro 5. Continuación.

\begin{tabular}{|c|c|c|c|c|c|c|}
\hline \multirow[b]{2}{*}{ Variable } & \multicolumn{3}{|c|}{ Lineal } & \multicolumn{3}{|c|}{ Logaritmos } \\
\hline & Convencional & Miope & Racional & Convencional & Miope & Racional \\
\hline$C_{\mathrm{t}+1}$ & & & $\begin{array}{c}0.0016 \\
(0.1817)\end{array}$ & & & \\
\hline $\ln C_{t-1}$ & & & & & $\begin{array}{l}0.3236^{* * *} \\
0.1016\end{array}$ & $\begin{array}{c}0.0462 \\
(0.1743)\end{array}$ \\
\hline $\ln \mathrm{C}_{\mathrm{t}+1}$ & & & & & & $\begin{array}{c}-0.0829 \\
(0.1979)\end{array}$ \\
\hline Cons & $\begin{array}{l}-11.7917 \\
(41.7345)\end{array}$ & $\begin{array}{c}-22.5390 \\
(40.4347)\end{array}$ & $\begin{array}{c}-18.7162 \\
(39.039)\end{array}$ & $\begin{array}{c}-1.1526 \\
(1.6403)\end{array}$ & $\begin{array}{c}-1.5947 \\
(1.7477)\end{array}$ & $\begin{array}{c}-1.4618 \\
(1.5953)\end{array}$ \\
\hline $\mathrm{R}^{2}$ ajust. & 0.5048 & 0.5056 & 0.4718 & 0.4855 & 0.6611 & 0.5112 \\
\hline $\begin{array}{l}\text { Breusch- } \\
\text { Pagan } \chi^{2}\end{array}$ & 0.05 & 0.02 & 0.01 & $9.52^{* * *}$ & $8.39 * * *$ & $6.20 * * *$ \\
\hline $\begin{array}{l}\text { Breusch- } \\
\text { Godfrey } \chi^{2}\end{array}$ & 1.6 & 0.63 & 0.55 & 0.57 & $3.27^{*}$ & 0.08 \\
\hline $\mathrm{N}$ & 78 & 77 & 76 & 78 & 77 & 76 \\
\hline
\end{tabular}

Notas: Véase cuadro 3 para los nombres de las variables. La variable dependiente es el consumo de tabaco (Q). Los errores estándar se presentan entre paréntesis. $\mathrm{R}^{2}$ es el coeficiente de determinación, $\mathrm{N}$ representa el número de observaciones empleadas para estimar cada modelo. ${ }^{*} \mathrm{p}$-valor $<0.10,{ }^{* *} \mathrm{p}$-valor $<0.05, * * * \mathrm{p}$-valor $<0.01$. In representa logaritmo natural. La hipótesis nula en la prueba Breush-Pagan es que la varianza de los errores es constante (homoscedásticos). La hipótesis nula en la prueba Breusch-Godfrey es que no existe correlación serial (no-autocorrelación).

Fuente: Estimaciones propias. 


\section{Cuadro 6. Estadísticos de prueba de endogeneidad y validez de instrumentos para los modelos de adicción miope y adicción racional}

\begin{tabular}{|c|c|c|c|c|c|}
\hline & & \multicolumn{2}{|c|}{ Modelo de adicción miope } & \multicolumn{2}{|c|}{ Modelo de adicción racional } \\
\hline Instrumentos & Especificación & $\begin{array}{c}\text { Prueba de } \\
\text { Hausman }\left(\chi^{2}\right)\end{array}$ & $\begin{array}{c}\text { Validez de } \\
\text { instrumentos }\end{array}$ & $\begin{array}{c}\text { Prueba de } \\
\text { Hausman }\left(\chi^{2}\right)\end{array}$ & $\begin{array}{c}\text { Validez de } \\
\text { instrumentos } \\
\left(x^{2}\right)\end{array}$ \\
\hline \multirow{2}{*}{$\begin{array}{l}\text { Dos rezagos precio } \\
\text { tabaco }\end{array}$} & Logarítmico & $\begin{array}{c}2.1051 \\
(0.1515)\end{array}$ & $\begin{array}{l}5.1848 \\
(0.0228)^{* *}\end{array}$ & $\begin{array}{l}3.2888 \\
(0.0438)^{* *}\end{array}$ & - \\
\hline & Lineal & $\begin{array}{l}4.2123 \\
(0.0441)^{* *}\end{array}$ & $\begin{array}{l}9.5193 \\
(0.0020)^{* * *}\end{array}$ & $\begin{array}{l}8.3946 \\
(0.0006)^{* * *}\end{array}$ & - \\
\hline \multirow{2}{*}{$\begin{array}{l}\text { Rezago y adelanto } \\
\text { precio tabaco }\end{array}$} & Logarítmico & $\begin{array}{c}0.2960 \\
(0.5882)\end{array}$ & $\begin{array}{l}16.9400 \\
(0.0000)^{* * *}\end{array}$ & $\begin{array}{l}9.4071 \\
(0.0003)^{* * *}\end{array}$ & - \\
\hline & Lineal & $\begin{array}{c}1.4765 \\
(0.2287)\end{array}$ & $\begin{array}{l}22.3035 \\
(0.0000) * * *\end{array}$ & $\begin{array}{l}16.4903 \\
(0.0000)^{* * *}\end{array}$ & - \\
\hline \multirow{2}{*}{$\begin{array}{l}\text { Dos rezagos y dos } \\
\text { adelantos precio } \\
\text { tabaco }\end{array}$} & Logarítmico & $\begin{array}{c}0.0719 \\
(0.7894)\end{array}$ & $\begin{array}{l}21.9215 \\
(0.0001)^{* * *}\end{array}$ & $\begin{array}{c}0.1331 \\
(0.8757)\end{array}$ & $\begin{array}{l}21.4114 \\
(0.0000)^{* * *}\end{array}$ \\
\hline & Lineal & $\begin{array}{c}0.5201 \\
(0.4734)\end{array}$ & $\begin{array}{l}25.4699 \\
(0.0000)^{* * *}\end{array}$ & $\begin{array}{c}2.3058 \\
(0.1082)\end{array}$ & $\begin{array}{l}15.7105 \\
(0.0004)^{* * *}\end{array}$ \\
\hline \multirow{2}{*}{$\begin{array}{l}\text { Dos rezagos precio } \\
\text { tabaco y IEPS }\end{array}$} & Logarítmico & $\begin{array}{c}2.2346 \\
(0.1397)\end{array}$ & $\begin{array}{l}8.1386 \\
(0.0171)^{* *}\end{array}$ & $\begin{array}{l}4.6039 \\
(0.0136)^{* *}\end{array}$ & $\begin{array}{c}0.2662 \\
(0.6059)\end{array}$ \\
\hline & Lineal & $\begin{array}{l}5.5838 \\
(0.0181)^{* *}\end{array}$ & $\begin{array}{l}10.0850 \\
(0.0065)^{* * *}\end{array}$ & $\begin{array}{c}2.4438 \\
(0.0950)^{*}\end{array}$ & $\begin{array}{c}3.2881 \\
(0.0698)^{*}\end{array}$ \\
\hline \multirow{2}{*}{$\begin{array}{l}\text { Dos adelantos precio } \\
\text { tabaco y IEPS }\end{array}$} & Logarítmico & $\begin{array}{l}3.6594 \\
(0.0602)^{*}\end{array}$ & $\begin{array}{l}6.5770 \\
(0.0373)^{* *}\end{array}$ & $\begin{array}{l}6.3148 \\
(0.0032)^{* * *}\end{array}$ & $\begin{array}{c}0.2552 \\
(0.6135)\end{array}$ \\
\hline & Lineal & $\begin{array}{c}1.1345 \\
(0.2908)\end{array}$ & $\begin{array}{l}17.3920 \\
(0.0002) * * *\end{array}$ & $\begin{array}{c}1.2654 \\
(0.2892)\end{array}$ & $\begin{array}{c}2.0209 \\
(0.1551)\end{array}$ \\
\hline \multirow{2}{*}{$\begin{array}{l}\text { Rezago y adelanto } \\
\text { precio tabaco y IEPS }\end{array}$} & Logarítmico & $\begin{array}{c}0.4287 \\
(0.5149)\end{array}$ & $\begin{array}{l}18.4480 \\
(0.0001)^{* * *}\end{array}$ & $\begin{array}{c}2.0956 \\
(0.1314)\end{array}$ & $\begin{array}{l}5.6169 \\
(0.0178)^{* *}\end{array}$ \\
\hline & Lineal & $\begin{array}{c}1.7802 \\
(0.1867)\end{array}$ & $\begin{array}{l}23.1302 \\
(0.0000) * * *\end{array}$ & $\begin{array}{l}11.7186 \\
(0.0000)^{* * *}\end{array}$ & $\begin{array}{c}1.2343 \\
(0.2666)\end{array}$ \\
\hline \multirow{2}{*}{$\begin{array}{l}\text { Dos rezagos y dos } \\
\text { adelantos precio } \\
\text { tabaco y IEPS }\end{array}$} & Logarítmico & $\begin{array}{l}0.00815 \\
(0.9284)\end{array}$ & $\begin{array}{l}25.5168 \\
(0.0000)^{* * *}\end{array}$ & $\begin{array}{c}0.2130 \\
(0.8087)\end{array}$ & $\begin{array}{l}24.2017 \\
(0.0000)^{* * *}\end{array}$ \\
\hline & Lineal & $\begin{array}{c}0.9102 \\
(0.3436)\end{array}$ & $\begin{array}{l}27.4809 \\
(0.0000) * * *\end{array}$ & $\begin{array}{c}1.5999 \\
(0.2101)\end{array}$ & $\begin{array}{l}20.3257 \\
(0.0001)^{* * *}\end{array}$ \\
\hline
\end{tabular}

Notas: La hipótesis nula en la prueba de Hausman es que variables dependientes rezagada y/o adelantada, según sea el caso, son exógenas. En la prueba de validez de los instrumentos, la hipótesis nula es que el modelo está sobreidentificado. No rechazarla implica que los instrumentos son válidos. IEPS es el impuesto especial de producción y servicios al tabaco. P-valor en paréntesis. ${ }^{*}$ p-valor $<0.10,{ }^{* *}$ p-valor $<0.05, * * *$ p-valor $<0.01$.

Fuente: Estimaciones propias. 
ECONOMía TEORÍA y PRÁCTICA [ISSN: 2448-7481] - Nueva Época, año 27, número 51, julio-diciembre 2019, Jorge Ibarra Salazar, César González Caloca, Silvia Núñez Gómez, Manuel Ramírez García, Mario Rodríguez Nuncio

\section{Cuadro 7. Resultados de las estimaciones}

Modelo convencional:

Lineal: $C=\beta_{0}+\beta_{1}$ P $+\beta_{2}$ PIB $+\beta_{3}$ LGT $+\beta_{4}$ PUB $+\beta_{5}$ PIC $1+\beta_{6}$ PIC $2+\beta_{7}$ PIC 3

Logaritmos: $\ln C=\beta_{0}+\beta_{1} \ln \mathrm{P}+\beta_{2} \operatorname{lnPIB}+\beta_{3}$ LGT $+\beta_{4}$ PUB $+\beta_{5}$ PIC1 $+\beta_{6}$ PIC $2+\beta_{7}$ PIC 3

Modelo de adicción miope:

Lineal: $C=\beta_{0}+\beta_{1}$ P $+\beta_{2}$ PIB $+\beta_{3}$ LGT $+\beta_{4}$ PUB $+\beta_{5}$ PIC $1+\beta_{6}$ PIC $2+\beta_{7}$ PIC $3+\beta_{8} C_{t-1}$

Logaritmos: $\ln C=\beta_{0}+\beta_{1} \ln P+\beta_{2} \operatorname{lnPIB}+\beta_{3}$ LGT $+\beta_{4}$ PUB $+\beta_{5}$ PIC1 $+\beta_{6}$ PIC2 $+\beta_{7}$ PIC $3+\beta_{8} \ln C_{t-1}$

Modelo de adicción racional:

Lineal: $C=\beta_{0}+\beta_{1}$ P $+\beta_{2}$ PIB $+\beta_{3}$ LGT $+\beta_{4}$ PUB $+\beta_{5}$ PIC $1+\beta_{6}$ PIC $2+\beta_{7}$ PIC $3+\beta_{8} C_{t-1}+\beta_{9} C_{t+1}$ Logaritmos: $\ln C=\beta_{0}+\beta_{1} \ln P+\beta_{2} \ln$ PIB $+\beta_{3}$ LGT $+\beta_{4}$ PUB $+\beta_{5}$ PIC1 $+\beta_{6}$ PIC2 $+\beta_{7}$ PIC $3+\beta_{8} \ln C_{t-1}+\beta_{9}$ $\operatorname{lnC} \mathrm{C}_{\mathrm{t}+1}$

\begin{tabular}{|c|c|c|c|c|c|c|}
\hline \multirow[b]{2}{*}{ Variable } & \multicolumn{3}{|c|}{ Lineal } & \multicolumn{3}{|c|}{ Logaritmos } \\
\hline & Convencional $^{\mathrm{a}}$ & Miope $^{b}$ & Racional' $^{c}$ & Convencional $^{d}$ & Miope $^{d}$ & Racional $^{d}$ \\
\hline $\mathrm{P}$ & $\begin{array}{l}-0.7515^{* * *} \\
(0.2055)\end{array}$ & $\begin{array}{l}-0.8860^{* * *} \\
(0.2318)\end{array}$ & $\begin{array}{c}-1.1120^{*} \\
(0.5589)\end{array}$ & & & \\
\hline PIB & $\begin{array}{l}1.1609 * * * \\
(0.2836)\end{array}$ & $\begin{array}{l}1.3040^{* * *} \\
(0.3379)\end{array}$ & $\begin{array}{c}3.2389 * * \\
(1.2849)\end{array}$ & & & \\
\hline $\ln P$ & & & & $\begin{array}{l}-0.1030^{* *} \\
(0.0418)\end{array}$ & $\begin{array}{l}-0.0680 \\
(0.0503)\end{array}$ & $\begin{array}{l}-0.0791^{* *} \\
(0.0368)\end{array}$ \\
\hline InPIB & & & & $\begin{array}{l}1.2848^{* * *} \\
(0.3290)\end{array}$ & $\begin{array}{l}1.0309 * * * \\
(0.3611)\end{array}$ & $\begin{array}{l}1.3675^{* * *} \\
(0.4917)\end{array}$ \\
\hline LGT & $\begin{array}{c}6.8282 \\
(10.1767)\end{array}$ & $\begin{array}{c}7.1331 \\
(8.4346)\end{array}$ & $\begin{array}{c}-5.8444 \\
(19.2860)\end{array}$ & $\begin{array}{c}-0.0802 \\
(0.0659)\end{array}$ & $\begin{array}{c}-0.0361 \\
(0.0496)\end{array}$ & $\begin{array}{c}-0.0500 \\
(0.0702)\end{array}$ \\
\hline PUB & $\begin{array}{c}3.8235 \\
(6.8322)\end{array}$ & $\begin{array}{c}5.5673 \\
(9.0244)\end{array}$ & $\begin{array}{l}-13.9385 \\
(21.2694)\end{array}$ & $\begin{array}{c}-0.0478 \\
(0.0452)\end{array}$ & $\begin{array}{c}-0.0561 \\
(0.0617)\end{array}$ & $\begin{array}{c}-0.0774 * \\
(0.0444)\end{array}$ \\
\hline PIC1 & $\begin{array}{c}-2.3961 \\
(13.6722)\end{array}$ & $\begin{array}{l}-0.1231 \\
(9.5002)\end{array}$ & $\begin{array}{c}-76.4723^{*} \\
(42.9751)\end{array}$ & $\begin{array}{l}-0.1181 \\
(0.1275)\end{array}$ & $\begin{array}{l}-0.1328^{* *} \\
(0.0630)\end{array}$ & $\begin{array}{l}-0.1796 \\
(0.1355)\end{array}$ \\
\hline PIC2 & $\begin{array}{c}-22.1216^{* * *} \\
(8.2849)\end{array}$ & $\begin{array}{l}-24.7187 * * \\
(11.3280)\end{array}$ & $\begin{array}{r}-128.657^{* *} \\
(58.7440)\end{array}$ & $\begin{array}{l}-0.3113 * * * \\
(0.0683)\end{array}$ & $\begin{array}{l}-0.2266 * * * \\
(0.0746)\end{array}$ & $\begin{array}{l}-0.3621^{* * *} \\
(0.1005)\end{array}$ \\
\hline PIC3 & $\begin{array}{c}-26.4450^{*} \\
(15.2306)\end{array}$ & $\begin{array}{c}-27.0919 * \\
(14.6304)\end{array}$ & $\begin{array}{r}-191.140 * * \\
(89.5163)\end{array}$ & $\begin{array}{l}-0.4617^{* *} \\
(0.1891)\end{array}$ & $\begin{array}{l}-0.4215^{* * *} \\
(0.1012)\end{array}$ & $\begin{array}{l}-0.5545^{* *} \\
(0.2328)\end{array}$ \\
\hline$C_{t-1}$ & & $\begin{array}{c}-0.1789 \\
(0.1829)\end{array}$ & $\begin{array}{c}-1.0123 \\
(0.6337)\end{array}$ & & & \\
\hline
\end{tabular}


Cuadro 7. Continuación.

\begin{tabular}{|c|c|c|c|c|c|c|}
\hline \multirow[b]{2}{*}{ Variable } & \multicolumn{3}{|c|}{ Lineal } & \multicolumn{3}{|c|}{ Logaritmos } \\
\hline & Convencional $^{\mathrm{a}}$ & Miope $^{b}$ & Racional $^{c}$ & Convencional $^{d}$ & Miope $^{d}$ & Racional ${ }^{d}$ \\
\hline$C_{t+1}$ & & & $\begin{array}{c}-1.9498^{*} \\
(1.0566)\end{array}$ & & & \\
\hline $\ln C_{t-1}$ & & & & & $\begin{array}{l}0.3236^{* * *} \\
(0.1018)\end{array}$ & $\begin{array}{c}0.0462 \\
(0.1742)\end{array}$ \\
\hline $\ln C_{t+1}$ & & & & & & $\begin{array}{c}-0.0829 \\
(0.1978)\end{array}$ \\
\hline Cons & $\begin{array}{c}-11.7917 \\
(41.7345)\end{array}$ & $\begin{array}{c}-3.8655 \\
(47.7660)\end{array}$ & $\begin{array}{c}105.0585 \\
(118.4046)\end{array}$ & $\begin{array}{c}-1.1526 \\
(1.6406)\end{array}$ & $\begin{array}{c}-1.5947 \\
(1.7478)\end{array}$ & $\begin{array}{c}-1.4618 \\
(1.5951)\end{array}$ \\
\hline R2 ajust. & 0.5048 & 0.4362 & & 0.4855 & 0.6611 & 0.5112 \\
\hline $\mathrm{N}$ & 78 & 77 & 76 & 78 & 77 & 76 \\
\hline
\end{tabular}

Notas: Véase cuadro 3 para los nombres de las variables. La variable dependiente es el consumo de tabaco (C). Los errores estándar se presentan entre paréntesis. R2 es el coeficiente de determinación, $\mathrm{N}$ representa el número de observaciones empleadas para estimar cada modelo. ${ }^{*}$ significativo al $10 \%,{ }^{*}$ significativo al $5 \%$, ${ }^{* * *}$ significativo al $1 \%$. In representa logaritmo natural. a Estimado por mínimos cuadrados ordinarios. b Estimado por el método de variables instrumentales. Instrumentos utilizados: dos rezagos del precio. c Estimado por el método de variables instrumentales. Instrumentos utilizados: rezago y adelanto del precio, IEPS. d Estimado con errores estándar robustos para corregir heteroscedasticidad.

Fuente: Estimaciones propias.

\section{CONCLUSIONES}

En este artículo hemos estimado la demanda de tabaco en México de acuerdo con diferentes modelos de adicción, usando datos en series de tiempo trimestral desde el primer trimestre de 1994 y hasta el segundo trimestre de 2013. Nuestro objetivo ha sido analizar el efecto de la regulación de etiquetado y empaquetado en México, particularmente la implementada desde 2010 con las ASP sobre la demanda de tabaco.

Los estudios previos para México (Thrasher et al., 2013a; Thrasher et al., 2013b; Reid et al., 2013; Regalado et al., 2013; Thrasher et al., 2012a; Thrasher et al., 2012b; Thrasher et al., 2007; Thrasher et al., 2006) han notado la efectividad de las ASP en reducir las asimetrías de información y las externalidades negativas en la industria del tabaco, pero no han estimado su efecto sobre la demanda. Mientras el estudio de Thrasher et al. (2006) encuentra que los fumadores asignan un menor valor a los cigarros cuyas cajetillas contienen ASP e infieren que 
los pictogramas traerían como consecuencia una reducción en la demanda; en este trabajo hemos estimado la reducción en consumo de tabaco producida por esta medida regulatoria.

El principal hallazgo de la investigación es que la segunda y la tercera rondas de pictogramas causaron una reducción significativa y consistente en el consumo de tabaco, independientemente de la especificación y el modelo teórico adoptado. Igualmente consistente fue el resultado de la primera ronda, que no trajo un efecto significativo sobre el consumo de tabaco. Una explicación parcial de este resultado es que el efecto de los pictogramas en el consumo agregado se manifestó hasta la segunda ronda. Los resultados del modelo convencional, en su especificación en niveles, muestran que el consumo per cápita se redujo en 22 cigarrillos con la segunda ronda de pictogramas, y 26 cigarrillos con la tercera. Los efectos estimados a través del modelo de adicción miope, en su especificación logarítmica, indican que la segunda ronda causó una reducción en la demanda de $20.3 \%$ en el largo plazo, y de $14.2 \%$ en el corto plazo; en tanto que la tercera ronda redujo la demanda en $24.8 \%$ en el corto plazo y $34.4 \%$ en el largo plazo.

En la metodología se abordaron detalladamente todos los aspectos estadísticos que se pudieron presentar en la estimación de los modelos. Desde las características de las series de tiempo, hasta le estimación por mínimos cuadrados en dos etapas. Aunque creemos que de las estadísticas agregadas a escala nacional, la forma en que se cuantificó la demanda — producción de tabaco más importaciones- es la más adecuada, los resultados están condicionados a esta aproximación. Además de la variable independiente que se usó, también existen datos sobre las ventas de tabaco, tanto en unidades como en valor. El inconveniente de usar las ventas como aproximación de la demanda es que su comportamiento puede verse influido por las políticas de distribución e inventario de los fabricantes de productos de tabaco.

Por otro lado, si bien encontramos un efecto significativo de las ASP, el método empleado en esta investigación no indica en cuánto tiempo se manifiesta su efecto. Ése es un campo importante de futuras investigaciones.

\section{REFERENCIAS BIBLIOGRÁFICAS}

Ahsan, Abdillah, Wiyono, N. Hadi, Setyonaluri, Diahhadi; Denniston, Ryan, y So, Anthony D. (2014), "Illicit Cigarette Consumption and Government Revenue loss in Indonesia", Globalization and Health, vol. 10, núm. 75, pp. 01-08.

Appelbaum, Elie (1982), “The Estimation of the Degree of Oligopoly Power", Journal of Econometrics, vol. 19, pp. 287-299. 
Baltagi, Badi H., y Levin, Dan (1986), "Estimating Dynamic Demand for Cigarettes using Panel Data: The Effects of Bootlegging, Taxation and Advertising Reconsidered", The Review of Economics and Statistics, vol. 68, núm. 1, pp. 148-155. Bardsley, Peter, y Olekalns, Nilss (1999), "Cigarette and Tobacco Consumption: Have Anti-Smoking Policies Made a Difference?”, Economic Record, vol. 75, núm. 3, pp. 225-240.

Barnett, Paul G., Keeler, Theodore E., y Hu, The-Wei (1995), "Oligopoly Structure and the Incidence of Cigarette Excise Taxes”, Journal of Public Economics, vol. 57, núm. 3, pp. 457-470.

Becker, Gary S., y Murphy, Kevin M. (1988), "A Theory of Rational Addiction”, Journal of Political Economy, vol. 96, núm. 4, pp. 675-700.

, Grossman, Michael, y Murphy, Kevin M. (1994), "An Empirical Analysis of Cigarrette Addiction", The American Economic Review, vol. 84, núm. 3, pp. 396-418.

Bhuyan, Sanjib, y López, Rigoberto (1997), "Oligopoly Power in the Food and Tobacco Industries", American Journal of Agricultural Economics, vol. 79, pp. 1035-1043.

Cameron, Sam (1998), "Estimation of the Demand for Cigarettes: A Review of the Literature”, Economic Issues, vol. 3, núm. 2, pp. 51-71.

Chaloupka, Frank (1991), "Rational Addictive Behavior and Cigarrette Smoking", Journal of Political Economy, vol. 99, núm. 4, pp. 722-742.

Comisión Federal de Mejora Regulatoria, Cofemer (2012), Tabaquismo en México. Análisis y recomendaciones de mejora regulatoria, Cofemer, México.

Connelly, Richard T., Goel, Rajeev, y Ram, Rati (2009), "Demand for Cigarettes in the United States: Effects of Prices in Bordering States and Contiguity with Mexico and Canada", Applied Economics, vol. 41, núm. 18, pp. 2255-2260.

Cox, Howard, y Smith, Ron (1984), "Political Approaches to Smoking Control: A Comparative Analysis", Applied Economics, vol. 16, núm. 5, pp. 569-82.

Delipalla, Sophia, y O’Donnell, Owen (2001), "Estimating Tax Incidence, Market Power and Market Conduct: The European Cigarette Industry", International Journal of Industrial Organization, vol. 19, núm. 6, pp. 885-908.

Enders, Walter (2010), Applied Econometric Time Series, 3a ed., John Wiley \& Sons, Hoboken, Nueva Jersey.

Engle, Robert F., y Granger, Clive W. J. (1987), "Co-integration and Error Correction: Representation, Estimation, and Testing”, Econometrica, vol. 55, núm. 2, pp. 251-276.

Fujii, Edwin T. (1980), “The Demand for Cigarettes: Further Empirical Evidence and its Implications for Public Policy”, Applied Economics, vol. 12, núm. 4, pp. $479-489$. 
Goel, Rajeev K., y Morey, Mathew J. (1995), “The Interdependence of Cigarette and Liquor Demand”, Southern Economic Journal, vol. 62, núm. 2, pp. 451-459. , y Nelson, Michael (2006), “The Effectiveness of Anti-Smoking Legislation: A Review”, Journal of Economic Surveys, vol. 20, núm. 3, pp. 325-355. , y Nelson, Michael (2012), "Cigarette Demand and Effectiveness of U.S. Smoking Control Policies: State-level Evidence for more than Half a Century", Empirical Economics, vol. 42, núm. 3, pp. 1079-1095.

Guindon, G. Emmanuel, y Boisclair, David (2003), "Past, Current and Future Trends in Tobacco Use", HNP Discussion Paper, Economics of Tobacco Control Paper \#6, en línea. Consultado el 15 de octubre de 2018, en: http://siteresources.worl dbank.org/HEALTHNUTRITIONANDPOPULATION/Resources/28 1627-1095698140167/Guindon-PastCurrent-whole.pdf

Haden, Kim (1990), “The Demand for Cigarettes in Japan”, American Journal of Agricultural Economics, vol. 72, núm. 2, pp. 446-450.

Hamilton, James L. (1972), “The Demand for Cigarettes: Advertising, the Health Scare, and the Cigarette Advertising Ban", Review of Economics and Statistics, vol. 54, núm. 4, pp. 401-411.

Hammond, David (2009), Tobacco Labelling and Packaging Toolkit. A Guide to FCTC Article 11, en línea. Consultado el 16 de junio de 2015, en: http://www. tobaccolabels.ca/wp/wp-content/uploads/2013/12/IUATLD-Tookit-CompleteMar-3-2009.pdf

(2011), "Health Warning Messages on Tobacco Products: A Review", Tobacco Control, vol. 20, núm. 5, pp. 327-337.

Hausman, Jerry A. (1978), "Specification Tests in Econometrics”, Econometrica, vol. 46, pp. 1251-1271.

Houthakker, Hendrik S., y Taylor, Lester D. (1966), Consumer Demand in the United States, Analyses and Projections, Harvard University Press, Cambridge.

McLeod, Paul B. (1986), "Advertising Bans, Tobacco and Cigarette Consumption", Economic Letters, vol. 20, núm. 4, pp. 391-396.

Monárrez, Joel, Liu, Bojing, Greiner, Felix, Bremberg, Sven, y Galanti, Rosaria (2014), "Systematic Review of the Effect of Pictorial Warnings on Cigarette Packages in Smoking Behavior", American Journal of Public Health, vol. 104, núm. 10, pp. e11-e30.

Nguyen, Lien, Gunnar, Rosenqvist, y Pekurinen, Markku (2012), Demand for Tobacco in Europe. An Econometric Analysis of 11 Countries for the PPACTE Project, National Institute for Health and Welfare, Helsinki.

Noar, Seth M., Hall, Marissa G., Francis, Diane B., Ribisl, Kurt M., Pepper, Jessica K., y Brewer, Noel T. (2016), "Pictorial Cigarette Pack Warnings: A Meta-analysis of Experimental Studies”, Tobacco Control, vol. 25, núm. 3, pp. 341-54. 
Olivera, Rosa I., Cermeño, Rodolfo, Sáenz, Belén, Jiménez, Jorge A., y Reynales, Luz M. (2010), "El efecto del precio del tabaco sobre el consumo: un análisis de datos agregados para México", Salud Pública de México, vol. 52, núm. 2, pp. S197-S205. Organización Mundial de la Salud, OMS (2003), Convenio marco de la OMS para el control del tabaco, Ginebra, Suiza, WHO Document Production Services, en línea. Consultado el 6 de septiembre de 2015, en: http://www.who.int/fctc/es/ (2012), Informe mundial sobre los progresos realizados, Ginebra, Suiza, WHO, en línea. Consultado el 6 de septiembre de 2015, en: http://apps.who.int/iris/ bitstream/10665/79621/1/9789243504650_spa.pdf

Pekurinen, Markku (1989), “The Demand for Tobacco Products in Finland”, British Journal of Addiction, vol. 84, núm. 10, pp. 1183-1192.

Regalado, Justino, y Madrazo, Marcela (2013), “Adopción de advertencias sanitarias y su implementación: el caso de México", en Thrasher, J., Reynales, L., Lazcano, E., y Hernández, M. (eds.), Salud pública y tabaquismo, t. II. Advertencias sanitarias en América Latina y el Caribe, Instituto Nacional de Salud Pública, México, pp. 39-59.

, Hernández, Luis A., Cárdenas, Martha G., Robles, Ana M., Thrasher, James F., Arillo, Edna, y Pérez, Rosaura (2013), "Reacción de fumadores y no fumadores hacia las advertencias sanitarias más impactantes en el Distrito Federal, Guadalajara y Monterrey”, en Thrasher, J., Reynales, L., Lazcano, E., y Hernández, M. (eds.), Salud pública y tabaquismo, t. II, Advertencias sanitarias en América Latina y el Caribe, Instituto Nacional de Salud Pública, México, pp. 162-174. Reid, Jessica, Hammond, David, Thrasher, James F., Driezen, Peter, Boudreau, Christian, y Arillo, Edna (2013), "Impacto de estrategias visuales en las advertencias sanitarias: un experimento de campo en adultos fumadores y adolescentes mexicanos”, en Thrasher, J., Reynales, L., Lazcano, E., y Hernández, M. (eds.), Salud pública y tabaquismo, t. II, Advertencias sanitarias en América Latina y el Caribe, Instituto Nacional de Salud Pública, México, pp. 128-143.

Reynales, Luz M., Fleischer, Nancy L., Thrasher, James F., Zhang, Yiang, Meza, Rafael, Cummings, K. Michael, y Levy, David (2015), "Effects of Tobacco Control Policies on Smoking Prevalence and Tobacco-Attributable Deaths in Mexico: The SimSmoke Model", Revista Panamericana de Salud Pública, vol. 38, núm. 4, pp. 316-325.

, Thrasher, James F., Lazcano, Eduardo, y Hernández, Mauricio (eds.) (2013), Salud pública y tabaquismo, t. I, Políticas para el control del tabaco en México, Instituto Nacional de Salud Pública, México.

Schoenberg, E. H. (1933), “The Demand Curve for Cigarettes”, Journal of Business, vol. 6, núm. 1, pp. 15-35. 
Seldon, Barry J., y Doroodian, Khosrow (1989), "A Simultaneous Model of Cigarette Advertising: Effects on Demand and Industry Response to Public Policy", The Review of Economics and Statistics, vol. 71, núm. 4, pp. 673-677.

Stigler, George, y Becker, Gary S. (1977), "De Gustibus Non Est Disputandum”, American Economic Review, vol. 67, núm. 2, pp. 76-90.

Sung, Hai-Yen, Hu, The-Wei, y Keeler, Theodore E. (1994), "Cigarette Taxation and Demand: An Empirical Model”, Contemporary Economic Policy, vol. 12, núm. 3, pp. 91-100.

Thrasher, James F., Allen, Betania, Reynales, Luz M., Anaya, Rafael, Lazcano, Eduardo, y Herández, Mauricio (2006), "Análisis del impacto en fumadores mexicanos de los avisos gráficos en las cajetillas de cigarros", Salud Pública de México, vol. 48, núm. 1, pp. S65-S70.

, Allen, Betania, Rousu, M. C., Anaya, Rafael, Reynales, Luz M., Arillo, Edna, y Hernández, Mauricio (2007), "Estimating the Impact of Different Cigarette Package Warning Label Policies: The Auction Method", Addictive Behaviors, vol. 32, núm. 12, pp. 2916-2925.

, Arillo, Edna, Villalobos, Víctor, Pérez, Rosaura, Hammond, David, Sansores, Raúl, Valdemar, Fabiola, y Regalado, Justino (2013b), "Impacto de los contenidos en las advertencias sanitarias con pictogramas: un experimento de campo en adultos fumadores y adolescentes mexicanos", en Thrasher, J., Reynales, L., Lazcano, E., y Hernández, M. (eds.), Salud pública y tabaquismo, t. II, Advertencias sanitarias en América Latina y el Caribe, Instituto Nacional de Salud Pública, México, pp. 144-161.

, Pérez, Rosaura, Arillo, Edna, y Barrientos, Inti (2013a), "Evaluación del impacto de la primera ronda de advertencias sanitarias con pictogramas en México: resultados de dos encuestas en población fumadora", en Thrasher, J., Reynales, L., Lazcano, E., y Hernández, M. (eds.), Salud pública y tabaquismo, t. II, Advertencias sanitarias en América Latina y el Caribe, Instituto Nacional de Salud Pública, México, pp. 109-127.

, Pérez, Rosaura, Arillo, Edna, y Barrientos, Inti (2012a), "Impacto de las advertencias con pictogramas en las cajetillas de cigarros en México: resultados de una encuesta en fumadores de Guadalajara", Salud Pública de México, vol. 54, núm. 3, pp. 254-263.

, Allen, B., Reynales, Luz M., Anaya, Rafael, Lazcano, Eduardo, y Hernández, Mauricio (2006), "Análisis del impacto en fumadores mexicanos de los avisos gráficos en las cajetillas de cigarros”, Salud Pública de México, vol. 48, núm. 1, pp. s65-s74.

, Pérez, Rosaura, Arillo, Edna, y Barrientos, Inti (2012b), "Hacia el consumo informado de tabaco en México: efecto de las advertencias con pictogramas en población fumadora”, Salud Pública de México, vol. 54, núm. 3, pp. 242-253. 
U.S. National Cancer Institute, USNCI, y World Health Organization, WHO (2016), The Economics of Tobacco and Tobacco Control, National Cancer Institute Tobacco Control Monograph 21. NIH Publication, núm. 16-CA-8029A. Bethesda, MD, U.S. Department of Health and Human Services, National Institutes of Health, National Cancer Institute; and Geneva, $\mathrm{CH}$, World Health Organization. Waters, Hugh, Sáenz, Belén, Ross, Hana, y Reynales, Luz M. (2010), La economía del tabaco y los impuestos al tabaco en México, Unión Internacional contra la Tuberculosis y Enfermedades Respiratorias, París, en línea. Consultado el 15 de octubre de 2018, en http://www.who.int/fctc/reporting/party_reports/mexico_ annex2_economy_of_tobacco_and_taxes_in_mexico.pdf

Wilkins, Nick, Yurekli, Ayda, y Hu, The-Wei (2001), Economic Analysis of Tobacco Demand, World Bank Economics of Tobacco Toolkit, Washington, DC. 
